\title{
Infection and microleakage the caused of endodontic failure
}

\author{
Kurniasri Amas Achiar*, Gantini Subrata** \\ *Department of Conservative Dentistry Faculty of Dentistry Universitas Padjadjaran \\ **Department of Prosthodontics Faculty of Dentistry Universitas Padjadjaran
}

\begin{abstract}
The success of endodontic treatment depends on the quality of endodontic treatment and the final restoration. The mean reason for endodontic treatment failure is usually microleakage. That is why it becomes one of the priorities for dental research to prevent microleakage. Infection during the root canal treatment can be prevented. First, by employing strict aseptic clinical techniques follows by cleaning all bacteria and preoperative necrotic pulp-tissue remnants from the root canal. Irrigants are essential in this phase. Shaping of the canal is also an important prerequisite for endodontic success. Removal of the smear layer can enhance seal ability. Second, obturation of the root canal should leave the tooth in the most biological inert condition possible, and it must prevent reinfection as well as growth of any microorganisms remaining in the canal. The application of an antibacterial dressing between appointments is absolutely necessary or the root canal has to be obturate at the first appointment in order to deprive the microorganisms of nutrients and space to multiply. The temporary filling must be at least $3.5 \mathrm{~mm}$ thick. Failure occurs because of missed canals, iatrogenic events and radicular fractures have to be avoided. At the end clinicians have to confirm that the root canal is cleaned and hermetically obturated, because hermetic root canal filling will prevent the leakage of irritant to the apical area.
\end{abstract}

Key words: Infection, microleakage, endodontic failure

\section{INTRODUCTION}

In simple terms, success of a given therapy in medicine or dentistry may be defined as the result obtained which achieved the initial treatment goal. The term "failure" consequently may be defined as a treatment that did not reach the objective or fell short of the acceptance level. Undoubtedly, the major factors associated with endodontic failure are the persistence of microbial infection in the root canal system and/or the periradicular area. ${ }^{1}$ From a biological perspective, an endodontic failure is associated with an inflammatory process in the peri-radicular support structures of a tooth. Such a lesion is often evident in radiographs as an osteolytic bone lesion in the periapical region. The disease processes generally have a microbiogical etiology. Lesions of the pulp are often infectious, and treatments involve several critical steps that are aimed to restrain infections of the root canal system in teeth. While the critical steps to accomplish effective treatments from a microbiological point of view have been outlined and tested over many years of research, the acquired knowledge is often overlooked or disregarded. ${ }^{2}$ 
Microorganisms residing in the root canal system and less often, organisms in the periradicular tissue compartment support the pathological process. The lesion may have developed after endodontic treatment of a tooth with a vital pulp. The treatment failure is due, in the overwhelming number of cases, to an infectious process which has either been caused by a breach of asepsis during the treatment of a vital pulp or has resulted from lack of success in eliminating the existing root canal infection. Leakage of bacterial elements from the oral environment is another significant cause of endodontic treatment failure. ${ }^{3}$

Clinicians generally believe that endodontic procedural errors, such as underfilling, overfilling, separated instruments, root perforations, ledge formation etc, are the direct cause of endodontic treatment failure. However, procedural errors by themselves do not jeopardize the outcome of treatment unless a concomitant infection is present. ${ }^{4}$

Thus, the detection, management, and elimination of bacteria are of primary importance to dental clinician. Every root canal treatment procedure begins with a thorough diagnosis, including a complete clinical and radiographic examination. ${ }^{3}$ The increase in endodontic treatment would also increase the demand for restoring the endodontically treated teeth properly to function after the completion of endodontic treatment. Restoring the endodontically teeth is a further step after finishing endodontic treatment, to recover the physiologic and esthetic factor to a better level. Besides the quality of endodontic treatment itself, the success of endodontic treatment is depends on the final restoration. ${ }^{5,6}$

That is why it becomes one of the priorities for dental research to prevent microleakage. Despite many efforts to achieve this goal, the phenomenon continues to intrigue researchers. Research asserts that as of today all restorations badly suffer from microleakage irrespective of the material and restorative technique used. One of the fundamental goals of a dental treatment is to eradicate disease and restore the tooth to its "original" functions. Prosthodontist, endodontists, and dental materials specialists have been most innovative and hard working at introducing new treatment modalities, tooth/root preparation designs, and materials to achieve that goal. Techniques and materials capable of withstanding the most challenging conditions that may be present in the oral cavity have been successfully developed. Many of the problems in bringing tooth restoration to its "original" functions have been resolved. However, the great achievement of researchers continues to be threatened by one phenomenon. That phenomenon is micro leakage. $^{7}$

\section{Microleakage}

The mean reason for endodontic treatment failure is usually micro leakage. This phenomenon has also been described as marginal permeability, micro marginal leakage, fluid exchange, liquid diffusion, and capillary penetration. Microleakage is the result of the fact that current conservative materials do not chemically bond to cavity walls to the extent of forming a hermetic seal. Lack of a hermetic seal at the interface means a gap that allows the seepage of oral fluids between the restoration and the prepared tooth surface. Microleakage is probably the only phenomenon that can be singled out as the most harmful to conservative dental practice. Hundreds of scientific papers addressing the issue of microleakage have been published. Many of these articles used one of the numerous experimental procedures that compares sealing ability of a given tooth preparation design or a restorative material vs another. The data generally embodied terms such as "It appears that" material A or tooth preparation design A caused less leakage than that of $B$. Microleakage can occur in the root canal filling as well as in the coronal restoration system. $^{7}$

Microleakage is not the only cause of pulpal disease, but its deleterious effects have been clearly documented by many in vivo studies. The consequences of bacterial and toxin ingress can require many years to manifest. Actual bacterial penetration through obturating materials may not be necessary to cause treatment failure. More important maybe leakage of bacterial by-product. Bacterial metabolites, toxins, and degradation products are much smaller than bacteria and could penetrate faster. Therefore, any reduction in microleakage may support the pulpal defense system. ${ }^{8}$ Careful endodontic evaluation must be 
made before the dentist attempts to restore the endodontically treated tooth. Proper endodontic treatment with exact obturation of the root canal is the prerequisite for restoration of crownless teeth and teeth with minimal coronal tooth structure.

However, the coronal seal achieved by the restoration is considered as important for ultimate success of endodontic treatment. In this paper will be evaluated about infection during the root canal treatment and radicular microleakage, and in the second paper will be discussed about coronal microleakage. ${ }^{5,9}$

\section{Infection during the root canal treatment}

The aim of root canal treatment is to keep the tooth healthy or return the tooth to health, therefore allowing it to be functional. Root canals fail due to bacterial infection and microleakage. ${ }^{10}$ The primary cause of periradicular pathosis is bacterial infection in the root canal system. However, procedural errors also impedeendodontic therapy. There are two factors have been shown clearly to have a direct impact on the outcome of endodontic therapy, i.e. root canal infection at the time of root filling and a preoperative periradicular lesion. ${ }^{4,11}$

\section{Preventing root canal infection during root canal treatment}

Root canal treatment is necessitated by endodontic disease, which in turn, a product of bacteria. Thus, the detection, management, and elimination of bacteria are primary importance to the dental clinician. ${ }^{11}$ Every root canal treatment procedure begins with a thorough diagnosis, including a complete clinical and radiographic examination. In most cases, failure of endodontic treatment is a result of microorganisms persisting in the apical portion of the root canal system, even in well-treated teeth. Most of the studies have been describing microleakage usually happens from apical foramen to cervical third of the root canal and influences negatively on the success of endodontic therapy. ${ }^{1,10}$

There are several considerations of endodontic failures that must be prevented in connection with infection: Contamination should be prevented during the endodontic treatment by employing strict aseptic clinical techniques, which should always include the use of rubber dam. Using a rubber dam to keep the tooth absolutely dry is mandatory. ${ }^{12,13}$ But there is still widespread skepticism and reluctance among dentists to use the rubber dam. It is all about the time-consuming thing. Dentist must also aware of latex allergies while using rubber-dam.

Following that, treatment of the root canal systems consist of two phase: cleaning and shaping the access cavity to the canal.Cleaning The goal of root canal preparation is to clean all bacteria and a preoperative necrotic pulp-tissue remnants from the root canal. Cleaning refers to the removal of all contents of the root canal system. All caries must be remove before endodontic treatment to prevent bacterial contamination of the root canal which can caused secondary caries. Irrigants are essential when it comes to dissolving tissue, flushing bacteria, and cleaning the canal. Sodium hypochlorite is the most widely used, often in combination with ultrasonic activation, although other irrigants, such as chlorhexidine and electrolyzed oxidized water, have their benefits as well. $8,11,12$

Shaping. It refers to a specific cavity form with five objectives: develop a continuously tapering conical form in the root canal preparation, make the canal narrower apically, with the narrowest cross-sectional diameter at its terminus, make the preparation in multiple planes, keep the foramen as small as is practical that will allow the entire canal to be filled with a biological inert material. The basic mechanical instrumentation and shaping of the canal is the most important prerequisite for endodontic success. If the root canal is only partially instrumented, successful treatment can not be expected. As has been said explained above, the preparation should extend to the narrowest part of the root canal, the apical constriction. ${ }^{12}$

However, there are difficulties in length determination. The tooth length corresponds to the distance between the coronal reference point and the anatomic apex, while the working length is that between the coronal reference point and the apical constriction. The apical constriction however can not be definitely determined clinically. The preparation should end $0.5-1 \mathrm{~mm}$ short of the radiographic apex. This arbitrarily chosen endpoint should protect the apical foramen from being 
enlarged and help prevent bacteria from being forced into the periapical space. To be sure this length determination can leave masses of bacteria remaining in the critical apical zone so that a periapical lesion can develop after endodontic treatment. Therefore, to completely eliminate all bacteria and their products it makes sense to instrument all the way to the radiographic apex. Most obturation problems are really problems of cleaning and shaping. With the balance fore technique, significantly less dentin debris is push out through the apical foramen than with the step-back technique. ${ }^{12}$

The smear layer. Studies show that currently used methods of instrumentation, especially rotary instrumentation techniques, produce a smear layer that covers root canal walls and the opening to the dentinal tubules. The smear layer is formed on the root canal surface as the result of instrumentation, and closes the openings of the tubules. The smear layer consists of organic and inorganic substances, including fragments of odontoblastic processes, microorganisms, and necrotic materials. Presence of this smear layer prevents penetration of intracanal medication into the irregularities of the root canal system and the dentinal tubules and also prevents complete adaptation of obturation materials to the prepared root canal surfaces. ${ }^{14}$

How well the sealer adheres to the canal walls is also important. Smear layer can play a factor which may present sealer penetration into the dentinal tubules. The presence of the smear layer in the dentinal walls impended penetration of the sealers Absence of the smear layer enables consistently, root canal sealer penetration into dentinal tubules. ${ }^{5}$ Removal of the smear layer enhanced seal ability as evidence by increased resistance to bacterial penetration. The incidence of apical leakage was reduced in the absence of the smear and the adaptation of gutta-percha was improved no matter what obturation method was used later. ${ }^{15}$ Various organic acids, ultrasonic instruments and lasers have been used to remove the smear layer from the surface of instrumented root canals. It is relatively easy for bacteria to adhere to the smear layer. The removal of the smear layer with organic acid: $6 \%$ citric acid solution and a final irrigation with sodium hypochlorite reduces the number of bacteria by $15 \%$ as it is simultaneously cleans the canal wall. Irrigation is the key to removal of smear layer lining the canal walls. In addition to acids, ultrasonic, and lasers, tetracycline has been recommended as a chelating agent during periodontal and endodontic treatment. ${ }^{12,15}$

Obturation. The last step in root canal treatment is to create the best possible hermetic seal with a filling material that is nonirritating to the tissues. The purpose of root canal obturation is to avoid penetration of microorganisms and their toxins from the oral cavity. through the root canal into periradicular tissue at both the coronal and apical ends. ${ }^{16}$

The purpose of obturation phase of an endodontic therapy is two fold: to prevent microorganisms from re-entering the root canal system and to isolate any microorganisms that may remain within the tooth from nutrients in tissue fluids. ${ }^{15}$ Bacteria can still be present in the root canal after instrumentation is completed and can be responsible for the return of painful symptoms. The root canal filling should leave the tooth in the most biological inert condition possible, and it must prevent reinfection as well as growth of any microorganisms remaining in the canal. But it must always be remembered that success will only be achieved if the root canal system has been as thoroughly cleaned as possible of infected material. ${ }^{5,12}$ After the cleaning and shaping or chemo mechanical instrumentation procedures of endodontic treatment, obturation of the root canal system should entomb persisting microorganisms that remain within infected dentinal tubules or within fins, cul-de-sac, etc. additionally, the root filing should completely seal the canal system from reinfection of tissue fluids. ${ }^{13}$ The ideal root canal filling material would be nonirritating to the pulp tissue, would tightly seal the canal both laterally and vertically and would be dimensionally stable so as not to shrink within the canal. It should not support bacterial growth and should even be bacteriostatic, while at the same time being biologically compatible and nontoxic. The filling material should be easy to sterilize before use. A root canal sealer should not harden to quickly and after hardening should exhibit good adhesion to both the dentin and the 
root canal filling. It should be insoluble in tissue fluids and have a slight expansion. ${ }^{11}$

\section{Radicular microleakage}

Microorganism persists in the root canal at the time of root filling or if they penetrate into the canal after filling, which is caused by microleakage. It has been described that apical microleakage can take place in the interface between sealer and the dentin, sealer and guttapercha, among the endodontic sealer itself or by its dissolution. ${ }^{16}$

Endodontic sealers continue to represent an important role for the control of microleakage, as they drain to the ramifications and improve adaptation of the filling to the irregularities of the interface between dentinal walls and guttapercha. Penetration of root canal sealers into the dentinal tubules could improves sealing of the root canal system. Such tubular penetration increases the interface between filling material and dentinal walls. Future trends idealized these materials filling out dentinal tubules, binding intimately to organic and inorganic interfaces of dentin, destroying or neutralizing microorganisms and their products, inducing cement neoformation and strengthening root canal system. This, in terms, may improve prevention of apical micro leakage by filling techniques. Furthermore retention of filling material might be improved by this mechanical interlocking. ${ }^{5,9}$

In the root canal treatment, root canal sealers serve to fill voids and minor discrepancies of fit between the gutta-percha filling and the root canal wall. Although sealers can form closed adhesion to the root canal wall, none is able to bond to the gutta percha core material. Upon setting, shrinkage of the sealer allows the sealer to pull away from gutta percha core, leaving a micro gap through which bacteria may pass. Supported in an invitro study found that gutta-percha does not offer an effective barrier to crown-down leakage when expose to oral environment. Additional studies using gutta-percha and various sealers indicate that gutta percha will allow bacterial leakage.

However, although gutta percha and conventional sealers have been considered the gold standard of endodontic obturation, these materials cannot be relied on when there is leakage. This problem has been overcome with the newer resin alternatives. Use of an adhesive sealer can significantly sloworstop bacterial migration..$^{12,15}$

Temporary seal of the root canal system during or after treatment (interim dressing)

If the root canal is not filled with a disinfecting dressing between appointments, bacteria can multiply and approach the numbers present at the beginning of the root canal treatment. In clinical practice it is recommended either that an interim dressing containing an antibacterial agent is placed, or that the root canal be obturate at the first appointment in order to deprive the microorganisms of nutrients and space to multiply. The dressing must serve as a barrier in case there is coronal leakage, combat embedded microorganisms and prevent the entry of apical secretions into the instrumented root canal. Remaining bacteria that are not remove by the mechanical preparation and the antibacterial irrigating solutions can multiply and cause failure. Phenol compounds used in interim dressings result in bacteria free root canals in $70 \%$ of cases. Calcium hydroxide has an even better effect. Almost all root canals were already free of bacteria after the first appointment.

The application of an antibacterial dressing between appointments is absolutely necessary. An inadequate coronal seal is just as often the cause of failure as incomplete elimination of bacteria from the root canal. Therefore an impervious coronal filling must be inserted between each appointment as well as at the conclusion of the root canal treatment to prevent recontamination of bacteria. The temporary filing must be at least $3.5 \mathrm{~mm}$ thick. ${ }^{16}$

Failures occur because of missed canals, iatrogenic events, and radicular fractures

Accessory canals maybe present in the pulp chamber. Bacteria located in areas such as ramification may sometimes be unaffected by endodontic treatment procedures..$^{1,12}$ This maybe an additional source of leakage that often goes unaddressed either following obturation of the canals or during the restoration phase. Overlooked primary canals or apical ramifications are the most common causes of endodontic failure. Branches 
which are difficult to find with instruments, can be cleaned effectively by copious irrigation with sodium hypochlorite. Placement a layer of resinmodified glass ionomer cement or adhesive resin to seal this area immediately following obturation can prevent leakage prior to final restoration of the tooth. $13,15,17$

\section{latrogenic factors}

Broken instruments. Clinicians has to avoid this broken instruments thing while doing the root canal treatment. If the broken instruments left in the root canal and can not be removed, it can become a source of infection.

Perforation. Excessive initial enlargement can lead to penetration of canal wall. The path of the root canal must be carefully evaluated on the diagnostic radiograph before the decision to use Gates-Glidden drills is made. Hegstrom files up to size 25 can be use just as well for the initial enlargement of the canal. Excessive removal of dentin can be avoided by filling toward the curvature of the canal. ${ }^{12}$

Overinstrumentation. Enlargement of the apical constriction can result in postendodontic reactions. This danger can be reduced by accurate radiographic determination of the length with identification of the correct apical reference point, which can be different for every root canal. ${ }^{12}$

Overfilling. The toxicity of the root canal filling materials has been considered to play an important role. In most cases apical sealing is inadequate in overfilled root canals. Percolation of tissue fluid rich in glycoprotein into the root canal system can supply substrate to residual microorganisms, which can proliferate and reach sufficient number to induce or perpetuate a periradicular lesion. It is also well known that over instrumentation usually precedes overfilling. In teeth with infected necrotic pulps over instrumentation induces the displacement of infected dentine or debris into the periradicular tissues. The presence of infected dentine or cementum chips in the periradicular lesion has been associated with impaired healing. ${ }^{1}$

Underfilling. The root canal filling materials does not reach the proper working length, so that it creates space between the root filling material and the apical constgriction. The apical tissue fluid can reach the root canal.
Radicular fracture. The lost of tooth structure can have an adverse effect on the mechanical properties of a tooth. Thus it is the quantity of dentin remaining in the crown that largely determines the strength of endodontically treated teeth. However in most cases procedural errors do not jeopardize the outcome of endodontic treatment unless a concomitant infection is present. ${ }^{1,12}$

\section{CONCLUSION}

Regardless of the etiology, the sum of all causes is leakage, bacterial contamination or recontamination. Clinicians has to confirm that the root canal is cleaned and hermetically obturated, regardless of the obturation technique, because hermetic root canal filling will prevent the leakage of irritant to the apical area.

\section{REFERENCE}

1. Siqueira JF. An etiology of root canal treatment failure. why well-treated teeth can fail. Int Endod J 2001;34:1-10.

2. Spångberg L. Contemporary endodontology. Austr Endod J 1998;24:11-6.

3. Bergenholtz G, Spangberg L. Controversies in endodontics. Crit Rev Oral Biol Med 2004;15(2):99-114.

4. Louis M, Lin BDS, Rosenberg PA, Lin J. Do Procedural errors cause endodontic treatment failure. J Am Dent Assoc 2005;136(2):187-93.

5. Cheung W. Properties of and important concepts in restoring the endodontically treated teeth. Dent Asia 2004 Sept/Oct;40-7.

6. Ray HA, Trope M. Periapical status of endodontically treated teeth in relation to the technical quality of the root filling and the coronal restoration. Int Endod J 1995;28:128.

7. Al-Tahawi HM, Al-Shammery AR. Real time reference controlled reverse diffusion quantitation of microleakage: Justification. The Saudi Dent J 1995;7(3):167-76.

8. White SN, Yu Z, Tom, JFMD, Sangsurasak S. In vivo microleakage of luting cements for cast crowns. J Prosthet Dent 1994;71:333-8.

9. Segovic S, Galic N, Dvanzo A, Pavelic B. Post endodontic tooth restoration-Part I: The aim 
and the plan of the procedure. Acta Stomatol Croat 2004;38:1.

10. Ruddle CJ. Rationale for treatment evidencebased endodontic. Advanced endodontics. [cited 2008 March 1] Available from: http: / / www.endoruddle.com/contact. html?name=FAQConvTx.

11. De-Deus G, Gurgel-Filho ED, Magnilia-Ferreira C, Coutinho-Filho T. Influence of the filling technique on depth of tubular penetration of root canal sealer: A scanning electron microscopy study. Braz J Oral Sci 2004;3(9):4338.

12. Beer R, Baumann MA, Kim S. Color atlas of dental medicine: Endodontology. Thieme, Stuttgart. New York: Pliezhausen; 2000. p.107,127,199,210-1,272-3.

13. Rosenstiel SE, Land MF, Fujimoto J.
Contemporary fixed prosthodontics. $3^{\text {rd }}$ ed. USA: Mosby Inc.; 2001. p. 272-300.

14. Torabinejad M, Khademi AA, Babagoli J, Cho Y, Johnson WB, Bozhilov K, Kim J, Shabahang S. A new solution for the removal of the smear layer. J Endod 2003;29:3.

15. Kurtzman GM. Improving endodontic success through coronal leakage prevention. Dental Tribune Asia Pasific Edition; p. 20-23 [cited 2008 March1] Available from: http://www. gcamerica.com/images/pdfs/Kurtzman coronal leakage Triage.pdf.

16. Segovic S, Anic I, Stipetic-Ovcaricek J, Galic N, Pavelic B. Microleakage of post endodontic systems. Acta Stomatol Croat 2003;37:217-9.

17. Grossman LI, Oliet S, Del Rio CE. Ilmu endodontik dalam praktek. $11^{\text {th }}$ ed. Jakarta: Penerbit Buku Kedokteran EGC; 1995. 